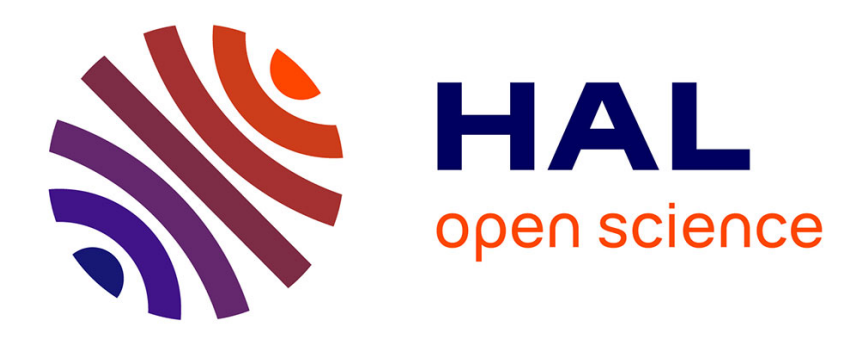

\title{
Product flexibility and price competition in Hotelling's duopoly
}

Pierre Fleckinger, Thierry Lafay

\section{To cite this version:}

Pierre Fleckinger, Thierry Lafay. Product flexibility and price competition in Hotelling's duopoly. Mathematical Social Sciences, 2010, 60 (1), pp.61-68. hal-00641873

\section{HAL Id: hal-00641873 \\ https://hal.science/hal-00641873}

Submitted on 16 Nov 2011

HAL is a multi-disciplinary open access archive for the deposit and dissemination of scientific research documents, whether they are published or not. The documents may come from teaching and research institutions in France or abroad, or from public or private research centers.
L'archive ouverte pluridisciplinaire HAL, est destinée au dépôt et à la diffusion de documents scientifiques de niveau recherche, publiés ou non, émanant des établissements d'enseignement et de recherche français ou étrangers, des laboratoires publics ou privés. 


\title{
Product Flexibility and Price Competition in Hotelling's Duopoly*
}

\author{
Pierre Fleckinger† Thierry Lafay ${ }^{\ddagger}$
}

November 2009

\begin{abstract}
In a Hotelling's duopoly with a general transportation cost function, we study competition through catalog: each firm chooses at the same time a price and a location. With simultaneous catalog offer, there is no equilibrium in pure strategies for high valuations of the consumers, while a Stackelberg equilibrium exists under mild conditions. The follower is better off than the leader, whose price is smaller: the location preemption effect is weaker than the price leadership effect. We obtain closed-form solutions for the linear and quadratic costs cases. Using these results, we discuss the nature of competition depending on the relative flexibility of products and prices.
\end{abstract}

\footnotetext{
${ }^{*}$ We would like to thank Jean-Pierre Ponssard for detailed comments on an earlier version.

${ }^{\dagger}$ Paris School of Economics, University of Paris 1. email: pierre.fleckinger@univparis1.fr. Centre d'Economie de la Sorbonne, 106/112 Boulevard de l'Hôpital, 75647 Paris Cedex 13.

‡PRISM-Sorbonne, University of Paris 1. email: tlafay@univ-paris1.fr. 17 rue de la Sorbonne, 75231 Paris.
} 
Key Words: horizontal differentiation, catalog competition, sequential moves.

JEL Classification: L13 D43 M3

\section{Introduction}

On most markets, the product choice is less flexible than the price policy. This explains why a reasonable modeling assumption is that price competition occurs only after the choice of products. But this is not always the case for at least two reasons. A first reason is related to the nature of the product: In some economic sectors, firms enjoy a large adaptability of their product. Standardization of intermediary inputs makes production highly flexible. For example, computer stores can change the characteristics of the computers they sell within one day. The service sector also usually exhibits extreme adaptability: for instance, online travel agencies' offers change virtually in a continuous fashion, both with regards to price and products offered. As a consequence, on such markets, the choice of product and the choice of price are simultaneous. A second reason is related to price rigidity: some firms appear as leaders (in time) concerning both product choice and prices, as they do not revise their price offer when other competitors enter the market. For example, each shop of a chain stores may face different independent markets but the firm chooses the same type of product on all markets and a unique pricing policy. Local competitors reacts to these offers while the converse is obviously not true. Agency situations often explain this inertia: an employee that work as a seller is not allowed to lower the price of the product it sells but must stick to the announced price. The use of national advertisement, too, partly explains the price stickiness of chains. In this pa- 
per we focus on these horizontally differentiated markets where competition takes place through catalog, a product-price couple (rigid) announcement ${ }^{1}$.

In his pioneering paper, Hotelling's main motivation was to construct a model in which price competition does not take a "winner-takes-all" form, but in which small changes in price only affects smoothly the quantity sold by a competitor. In his setting, the author shows that if firms choose first their locations, their will be a tendency towards homogeneity of the product-the so-called principle of minimum differentiation. It is somehow paradoxical that the idea of 'stability' in competition put forth by Hotelling is actually invalid in his model. Indeed, as pointed out by d'Aspremont et al. (1979), a pure strategy equilibrium does not exist because a price equilibrium fails to exist when products are too similar. D'Aspremont et al. show in turn that with quadratic transportation costs an equilibrium exists ${ }^{2}$. In this modified setting, the firms locate at the extreme points of the market and differentiation is maximal. Many authors have subsequently found mitigated results about differentiation in equilibrium (Economides, 1984; Hinloopen and van Marrevijk, 1999, among others). In our model, we obtain that differentiation is generically intermediate, and this does not depend on the shape of costs.

\footnotetext{
${ }^{1}$ We refer here to the informative dimension of catalog. Indeed each firm produces only one kind of product, and it is the information on that product and its price that matters in the catalog. Recently, Monteiro and Page (2008) have used the term catalog to refer to product line. They study equilibrium existence in a nonlinear pricing game.

${ }^{2}$ As pointed out by MacLeod (1985), this implies that the mass of indifferent consumers is zero, which restores equilibrium existence in price, thanks to continuity of the payoffs. Another direction to restore equilibrium existence is to allow for mixed strategy in price (see Dasgupta and Maskin (1986a,b) for a general treatment and applications), which can be interpreted as occurrence of sales (Osborne and Pitchik, 1987).
} 
While models of horizontal differentiation have been the subject of a vast literature, we focus on a topic that has not received enough attention: the sequentiality of product and price choices (see Prescott and Visscher (1977) for an early contribution). In most of the related literature, it is usually assumed that firms choose location first (possibly sequentially as in Tabuchi and Thisse (1995)) once and for all, then compete simultaneously in price (Neven, 1987; Lambertini, 2002; Götz, 2005; Economides et al., 2002). This is consistent with the view that the location is a geographical parameter, or with the view that the design of the product is far less flexible than its price. As far as we know, only few papers modify the assumption of last stage simultaneous price competition. Anderson (1987) studies a price leadership configuration: the firms choose sequentially the locations, then a Stackelberg competition in price takes place. His aim is to determine which firm chooses to be the price leader, the answer is that the second entrant is induced to be price leader, given the location chosen by the first entrant. Lambertini (1997) compare different timing and underlines the fact firms should play simultaneously in locations and sequentially in prices in an unconstrained Hotelling model with quadratic costs.

In this paper, we investigate situations where location and prices are necessarily chosen together. The first result concerning this "catalog" competition is that in general there does not exist an equilibrium in pure strategies when competition is simultaneous. However, in a sequential catalog competition, we are able to obtain the existence of subgame perfect equilibria with strictly positive profits for both firms under some assumptions. Contrary to traditional entry models, it is the follower that decides whether to exclude the incumbent or to accommodate, since only the follower firm has the ability to undercut its rival. The subgame perfect equilibrium is 
such that the leader (incumbent) always earns less than the follower ${ }^{3}$. This introduces a tension between the temptation of attrition - waiting to be the follower, which leads to an unstable configuration, and the will to guarantee a strictly positive profit.

Section 1 introduces the model and its assumptions. Section 2 gives the main insights concerning the general model and characterizes sequential catalog competition when a pure strategy subgame perfect equilibrium exists. We solve then the cases in which costs are linear (as in Hotelling's original model) and in which costs are quadratic. We also study the catalog competition in the circular model of Salop (1979). Section 3 contains a discussion of the model about sequentiality assumptions, examples are given where the results seem to have some predictive power. The concluding section sums up the results.

\section{The model}

We study a market where a mass 1 of consumers is distributed along the unit interval according to the density function $f$. A product choice corresponds to a point $x \in[0,1]$, the ideal product of the consumer located at $x$. We are interested in competition through catalog in the following sense: each firm chooses once and for all a product in $[0,1]$ and a price in $\mathbb{R}^{+}$. Firm $\mathrm{A}$ chooses $(a, p)$ and firm B chooses $(b, q)$. For convenience, we choose $a$ and $b$ as the distance of firms to zero ${ }^{4}$.

A consumer $x$ chooses to purchase a product according to the utility

\footnotetext{
${ }^{3}$ Note that if the incumbent is a chain store, this may be true at the local level, but overall, the chain store probably obtains a higher total profit (the sum over local retailers or franchisees) than a single local competitor.

${ }^{4}$ Note that in Hotelling (1929), b is the distance of product B to 1.
} 
maximization: Max $\{v-C(x-a)-p, v-C(b-x)-q, 0\}$. The reservation price is $v$ and a consumer buys a product only if the perceived price is less than his reservation price. The cost function $C$ is even, strictly increasing on the right of 0 , convex and twice continuously differentiable (except maybe at zero, where it admits a left and a right derivative). In Hotelling's original model, the cost function is simply the distance and consumers always consume the good, so that they minimize their disutility Min $(C(x-a)+p, C(b-x)+q)$. We mainly focus on the case in which $v$ is infinite. With or without a reservation price, consumer $x$ prefers firm B whenever $C(x-a)+p \geq C(b-x)+q$. We define the indifferent consumer, when unique, as $y$ such that $p+C(a-y)=q+C(b-y)$.

When a consumer is indifferent between firm A and B, we assume that it chooses the closest firm. Wherever this last criterion does not break indifference (when the distances to each firm are equal), we assume that some consumers choose A while others choose B. Formally, there exists some predetermined $\lambda$ with $0<\lambda<1$ such that firm A gets a share $\lambda$ and firm B a share $(1-\lambda)$ of those indifferent consumers. We first study the game where firms choose their catalog simultaneously, second we assume that firm A is a catalog leader. In this second setting, firm A first chooses its strategy $(a, p)$, then firm B chooses $(b, q)$ after having observed A's moves. Hence, we look for the subgame perfect equilibrium of the perfect information extensive game depicted in figure 1.

We may remark that if firm B chooses the same location as firm A, then its best response must be such that $q \leq p$ otherwise it gets no customer. In turn, by setting a slightly smaller price than firm A, firm B obtains the whole market. Thus if both firms choose the same location in equilibrium before choosing their price simultaneously, prices will be driven down to zero, 


\begin{tabular}{|c|c|c|}
\hline Firm A & Firm B & \\
\hline location $a$ & location $b$ & Sales realized \\
\hline
\end{tabular}

Figure 1: Sequential setting

following the logic of Bertrand competition. As pointed out by Anderson (1987, p. 379), Hotelling seems not to have well taken into account these "undercutting" strategies. In the sequential setting, as the first firm posts a price before the other enters, it cannot use such an undercutting strategy. On the contrary, firm B has a powerful threat with these undercutting strategies. The question is thus whether the leader can induce the follower not to undercut, thereby securing a positive profit.

\subsection{General results}

Combining the results of Hotelling and d'Aspremont et al., one gets the insights of the following result concerning catalog competition:

Proposition 1 For any distribution of the consumers', any convex cost function and an infinite willingness to pay, simultaneous catalog competition has no equilibrium in pure strategies.

A direct proof is rather intuitive ${ }^{5}$. First we notice that whatever the other firm's strategy, a firm can always get a strictly positive profit. Second, we remark that there cannot be an equilibrium where both firms choose the same product because then prices would be driven to zero. Let us therefore

\footnotetext{
${ }^{5}$ The results of Economides (1987) apply to the present setting, and we only sketch here the intuitions.
} 
assume that one firm is pricing less than the other. This firm has an incentive to choose the same product as its competitor, but then the other firm would make zero profit, thus it cannot be an equilibrium. The same argument holds when firms use the same price (except that firm will price slightly less than its competitor). Thus there is no Nash equilibrium in pure strategies when $v$ is infinite. However it is obvious that if $v$ is small, there are Nash equilibria as firms can enjoy local monopoly. This continues to hold for small enough $v$, in which case firms compete for some customers, but there is some finite level of $v$ beyond which pure strategy equilibrium does not exist any more ${ }^{6}$.

We now study the extensive game with sequential catalog issuing. We are able to state the following observation.

Proposition 2 For any distribution of the consumers', any cost function and any willingness to pay, the follower makes a (weakly) higher profit than the leader in any equilibrium of the sequential catalog competition.

This proposition is proved in appendix. It is of course straightforward if the leader cannot prevent undercutting ${ }^{7}$, since the leader makes then zero profit. This proposition shows that there is always a second mover advantage in this setting. ${ }^{8}$. In other words, the price followership effect is stronger than the location leadership effect in our model. This statement departs from the result in Anderson (1987). He studies the case in which firms sequentially

\footnotetext{
${ }^{6}$ In a related paper, we studied this point in details, see Fleckinger and Lafay (2003).

${ }^{7}$ For example, when the distribution boils down to a single atom, Bertand competition for this atom obtains, and there is a continuum of equilibria in which the leader makes zero profit.

${ }^{8}$ For a full analysis of first versus second mover advantage in the case of unidimensional strategies, see Gal-Or (1985).
} 
choose their location before sequentially choosing their prices. With that different timing structure he shows that a leader in location and price earns more than its follower.

This proposition also underlines that even when there exists an equilibrium in simultaneous catalog competition (which requires a finite $v$ as already mentioned), both firms are better off with sequential catalog competition. Indeed, the leader can always get as much profit as in the simultaneous game by choosing the equilibrium product and price of the simultaneous game. Formally, let $\Pi_{S}$ be the largest profit among the simultaneous Nash equilibria in pure strategies ${ }^{9}$ then $\Pi_{B} \geq \Pi_{A} \geq \Pi_{S}$. However, even if both firms earn more in the sequential setting, they both prefer to be the follower. In other words, they can agree to play a catalog competition sequentially but none wants to publish its catalog first, creating an attrition temptation.

From now on, feminine pronouns will refer to the leader and masculine ones to the follower. For a better understanding of the general shape of equilibrium (when it exists), a classification of the follower's strategies is in order. In a sequential catalog competition, he has two kinds of strategies. Either he chooses an exclusion strategy, which means that he sets a catalog such that he serves the whole market. Or he chooses an accommodation strategy such that the leader keeps a strictly positive market share. Among the possible exclusion strategies, the best one is clearly to choose the same product as the leader but with a slightly lower price, $p-\epsilon$, with $\epsilon$ as small as desired. In order to secure a positive market share, the leader must thus guarantee a profit at least equal to $p$ to the follower when using an accommodation strategy. We may note that contrary to the classical literature

\footnotetext{
${ }^{9}$ When they exist.
} 
about entry, in a catalog competition it is the follower who chooses between accommodation and exclusion.

Consider the point of view of the follower, for a given strategy of the leader $(a, p)$, with $a<\frac{1}{2}$ as it is pictured in Figure 2. The case $a \geq \frac{1}{2}$ happens to be similar, with symmetric considerations each time. For any price $q<p$, i.e. in zones I and IV, it is clear that the best reply is to undercut the leader, by setting $b=a$ and $q=p-\epsilon$. Any location in zone II is dominated by the point in zone III such that the location is symmetric with respect to $a$, keeping the same price, because then the market share is strictly bigger. It also holds for any catalog $(b, q)$ in II' such that the follower has a positive market share. The cases where the follower has zero market shares is obviously uninteresting. Overall, to find the best-reply of the follower, we can restrict our attention on one hand to the subspace where $b \geq a$ and $q>p$ (accommodation), and on the other hand to the best undercutting strategy (exclusion).

Now, in zone III and III', for any price $q$, the follower is better off choosing a catalog along the perceived price curve of the leader $(x, C(a-x)+p)$ , because it maximizes his market share. To see this, just notice that by definition of $y$, the market share of the follower is the mass of consumers to the right of $y$. Thus, for a constant price $q$, the goal of the follower is to minimize $y$. This is formally expressed in the next lemma which is proved in appendix.

Lemma 1 For any distribution of the consumers', any cost function and any willingness to pay, the follower chooses a catalog such that $y=b$ and $q>p$ in an equilibrium with accommodation. 


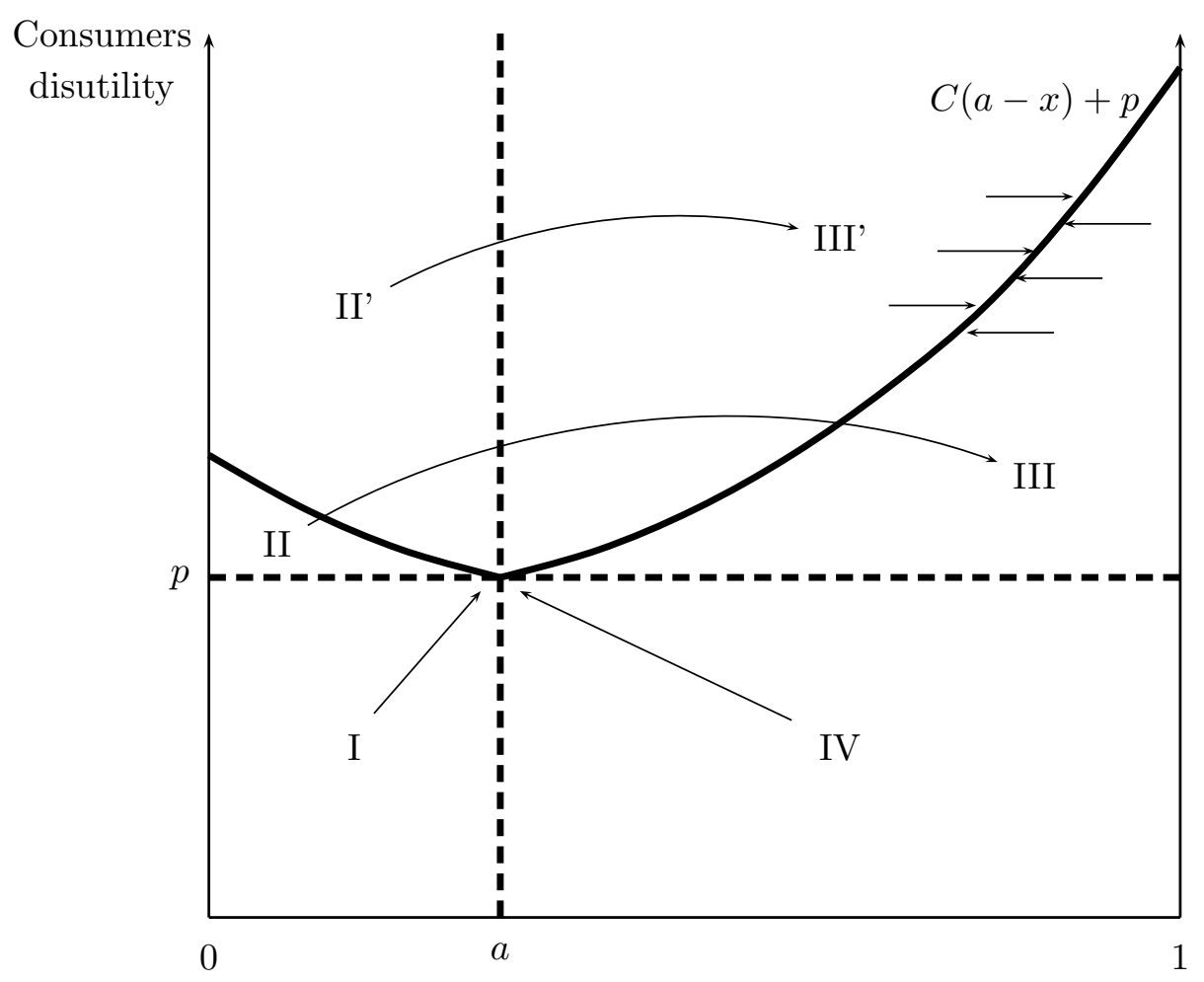

Figure 2: Strategies for the follower

Here the first mover is also the 'leader' in price (as she posts the lowest price). Indeed, the leader defines a price schedule and the follower uses this price umbrella to fix its price. It also departs from Bertrand competition where the follower in time is the leader in price.

We now investigate equilibrium existence. The main difficulty is related to continuity. First, even the existence results of Dasgupta and Maskin (1986a,b) for mixed strategy equilibrium do not apply because of a lack 
of upper hemi-continuity of the follower profit function ${ }^{10}$. Second, even though we now know that the follower chooses a catalog $(b, q)$ such that the indifferent consumer is $b$, the best-reply $b^{*}(a, p)$ may be a multivalued correspondence $^{11}$. Classically, this non uniqueness of best reply would imply a multiplicity of subgame perfect equilibria depending on the anticipation made by the leader regarding the way the follower will react. Third, the profit of firm A may not be a continuous function of $(a, p)^{12}$. To rule out these situations, we impose the following sufficient condition for uniqueness of the best reply of firm B.

Condition $1 C^{\prime \prime}(x)<2 C^{\prime}(x)$ for all $x>0$.

This condition imposes an upper bound on the relative curvature of the cost function. We are now in position to state the central result of this section.

Proposition 3 With uniform consumers' distribution and, under condition 1, there exists a subgame perfect equilibrium in a sequential catalog competition such that the leader makes a positive profit.

\footnotetext{
${ }^{10}$ For example the function $\Pi_{B}$ is such that $\lim _{\substack{q \rightarrow p \\ q<p}} \Pi_{B}(a, q)=p>(1-\lambda) p=\Pi_{B}(a, p)$. To overcome this issue, one can think of setting $\lambda=0$ but it would only shift the continuity problem to the leader's optimization.

${ }^{11}$ For example, consider the following convex, twice differentiable function: $C(x)=\left\{\begin{array}{ll}\frac{x}{1-x} & \text { if } x \leq \frac{1}{2} \\ 8 x^{2}-4 x+1 & \text { otherwise }\end{array}\right.$. For $a=0$ and $p=1$ the accommodation best reply is not uniquely defined as any $b \in\left[0, \frac{1}{2}\right]$ (associated with the corresponding $q=1+C(b)$ ) gives the same profit to the follower.

${ }^{12}$ Consider again the example of footnote 11, and fix $a=0$. For $p<1$, the follower's best reply is some $b \geq \frac{1}{2}$, for $p=1$, there is a continuum of best-replies, for $p>1$, exclusion is the unique best-reply. Therefore the profit of firm $\mathrm{A}$ is not continuous at $(a, p)=(0,1)$.
} 
The proof proceeds in four steps, and the sketch is as follows. First, we obtain the best reply of the follower if he uses an accommodation strategy. The leader has to choose her catalog in order to prevent an exclusion strategy. In turn, this implies that $p$ must be bounded. Finally, the leader maximizes a continuous function over a compact, which guarantees the existence of a solution. Of course, there might be equilibria even when condition 1 is not fulfilled as we will show: the quadratic model possesses a subgame perfect equilibrium obtained by backward induction.

\subsection{Linear cost (à la Hotelling)}

Following Hotelling's original assumption, we consider linear costs: $C(x)=$ $t|x|$, and look for the subgame perfect equilibrium of sequential catalog competition. Note that the conditions for equilibrium existence of proposition 2 are met. Hence, we follow the lines of the preceding proof to obtain the equilibrium. The optimal limit strategy of firm B if he decides to exclude the leader is such that $\left(b^{E x}, q^{E x}\right)=(a, p)$, implying $\Pi_{B}^{E x}(a, p)=p$. As the problem is symmetric regarding locations we compute the equilibrium such that $a \leq \frac{1}{2}$.

We begin by computing firm B's best reply if he decides to accommodate. From lemma 1, the optimal accommodation strategy is such that $q=p+$ $t(b-a)$. Therefore, firm B maximizes $\Pi_{B}^{A c}=(p+t(b-a))(1-b)$ s.t. $0 \leq a \leq b \leq 1$ and $p \geq 0, q \geq 0$ (these constraints, that define the valid domain, are omitted in the following). This profit is a concave function, and the first order condition implies: $\left(b^{A c}, q^{A c}\right)=\left(\frac{1+a-p / t}{2}, \frac{1-a+p / t}{2}\right)$. The solution has to be interior under the exclusion constraint $\Pi_{B}^{E x} \leq \Pi_{B}^{A c}$ since $\Pi_{B}^{A c}(b=1)=0$ and $\Pi_{B}^{A c}(b=a)<\Pi_{B}^{E x}$. Substituting the interior solution, 
we obtain $\Pi_{B}^{A c}(a, p)=\frac{(1-a+p / t)^{2}}{4}$ and $\Pi_{A}^{A c}(a, p)=\frac{1+a-p / t}{2} p / t$. Firm A seeks to maximize $\Pi_{A}^{A c}(a, p)$ under the constraint $\Pi_{B}^{A c}(a, p)=\frac{(1-a+p / t)^{2}}{4} \geq p$.

For any $p, \Pi_{A}^{A c}$ is increasing while $\Pi_{B}^{A c}$ is decreasing in $a$, thus the constraint must be binding. Substituting in the objective function, A's program boils down to maximizing $\frac{p}{t}\left(1-\sqrt{\frac{p}{t}}\right)$ for $p>0$. The value of the equilibrium parameters are in turn fully determined by using binding constraint and the expression of the best-reply. This allows us to state:

Proposition 4 The subgame perfect equilibrium of the linear cost game is such that (up to geometrical symmetry):

$$
\begin{gathered}
\left(a^{*}, p^{*}, b^{*}, q^{*}\right)=\left(\frac{1}{9}, \frac{4 t}{9}, \frac{1}{3}, \frac{2 t}{3}\right) \\
\Pi_{A}^{*}=\frac{4 t}{27} \quad \text { and } \quad \Pi_{B}^{*}=\frac{4 t}{9}
\end{gathered}
$$

We may notice that the distance between the firms is such that no equilibrium with competitive price exists. It violates the existence condition given by d'Aspremont et al. (1979). In other words, if the firms were able to revise (only) their prices, profits would be driven to zero by a price war. A price commitment of the leader is therefore valuable for both firms, because it allows to avoid an unstable configuration leading to zero profits. The locations and prices at equilibrium are depicted in figure 3. Of course the symmetric equilibrium $\left(a^{*}, p^{*}, b^{*}, q^{*}\right)=\left(\frac{8}{9}, \frac{4 t}{9}, \frac{2}{3}, \frac{2 t}{3}\right)$ is also possible.

\subsection{Quadratic cost (à la d'Aspremont et al.)}

In the quadratic cost case, if firms first choose locations (either sequentially or simultaneously) prior to prices, there is maximal differentiation (d'Aspremont et al., 1979). Firms price at $t$ and profits are then $t / 2$. 


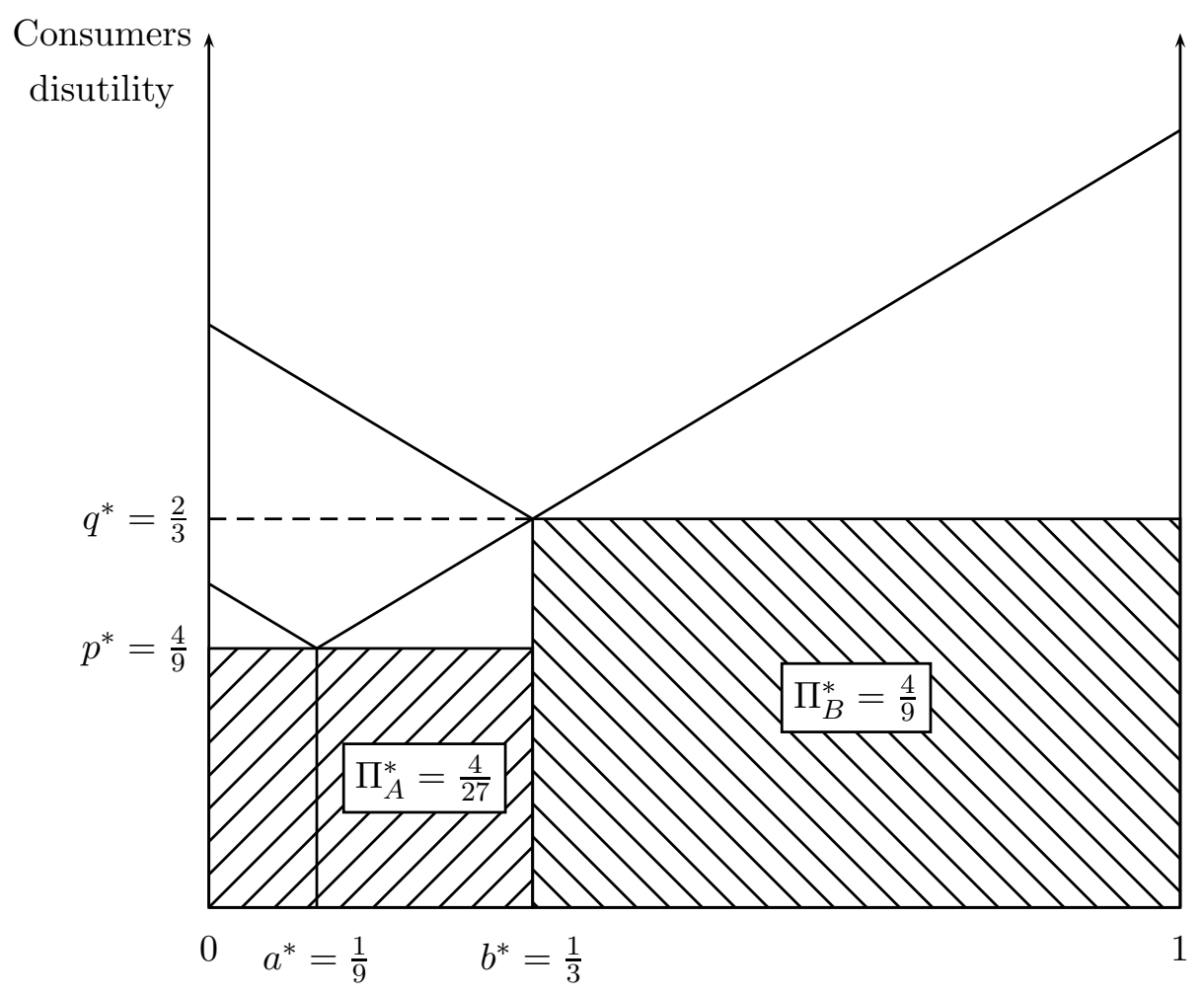

Figure 3: Equilibrium with linear costs for $t=1$

With quadratic costs, condition (1) of section 2 is not satisfied, and we cannot apply proposition 2 to claim existence of an equilibrium. However, we demonstrate in appendix that an equilibrium still exists by constructing it, and obtain:

Proposition 5 The subgame perfect equilibrium of the catalog competition in the quadratic cost game is such that (up to geometrical symmetry):

$$
\begin{aligned}
& \left(a^{*}, p^{*}, b^{*}, q^{*}\right)=\left(0, \frac{t}{4}, \frac{1}{2}, \frac{t}{2}\right) \\
& \Pi_{A}^{*}=\frac{t}{8} \quad \text { and } \quad \Pi_{B}^{*}=\frac{t}{4}
\end{aligned}
$$




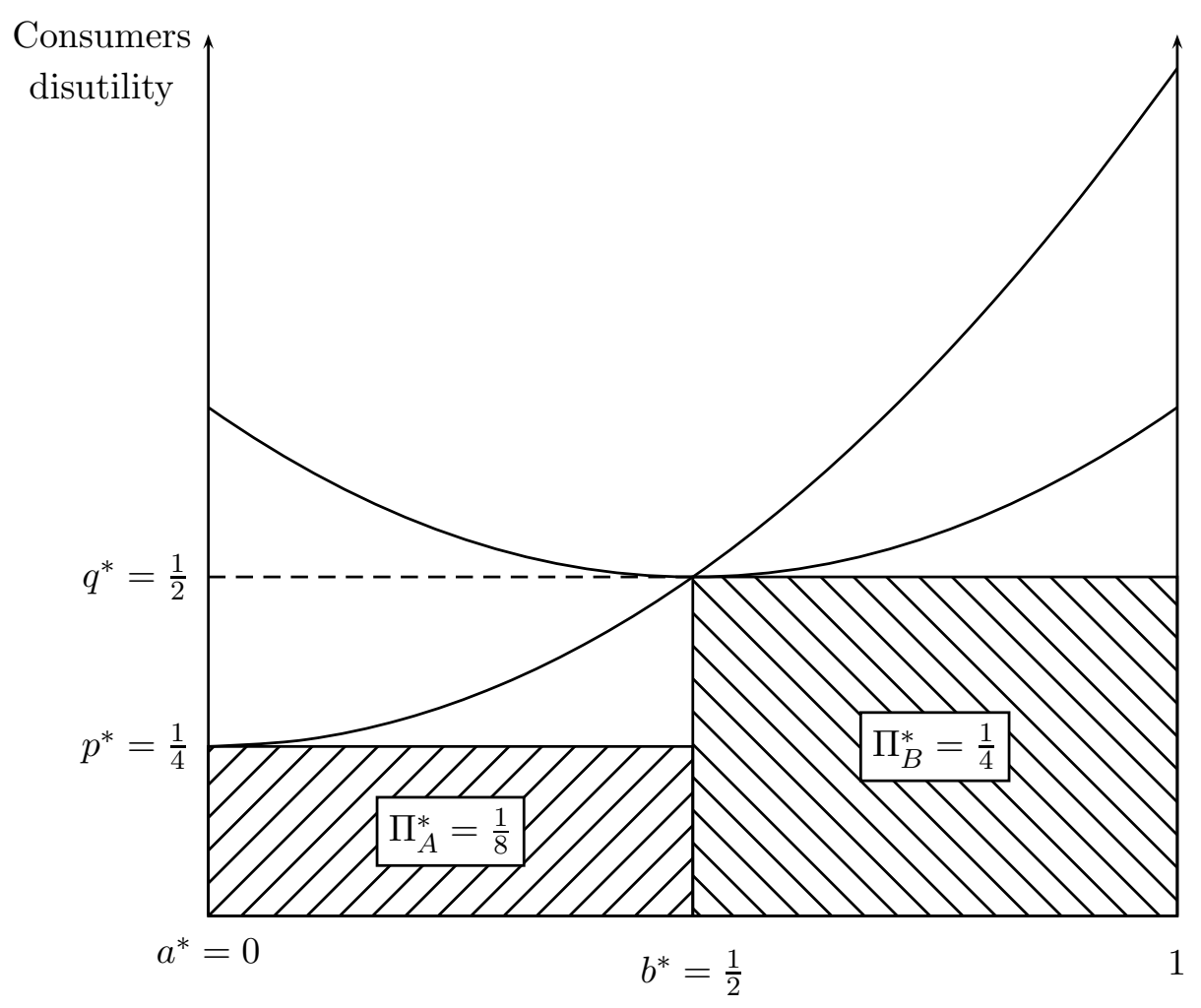

Figure 4: Equilibrium with quadratic costs for $t=1$

Thus, differentiation is more important with quadratic costs than linear ones. The leader chooses a niche, while the follower chooses a generic product. The locations and prices at equilibrium are depicted in figure 4 .

\subsection{Catalog competition in Salop's model}

We investigate here how the catalog competition operates on a circular market such as Salop (1979). There is a unit mass of consumers uniformly distributed along a circle. Indeed, it is easy to show that simultaneous catalog competition exhibits no equilibrium in pure strategies. The sequential catalog competition is such that the follower earns more than the leader 
and prices higher. However, contrary to Hotelling's model, in Salop's model there exists an equilibrium if both firms choose first a location (either simultaneously or sequentially ${ }^{13}$ ) then compete simultaneously in price. This equilibrium is such that firms choose opposite locations and price at $t$, so that their profits are $t / 2$. The question arises whether a sequential cata$\log$ competition is likely to appear. In order to answer this question, we investigate the equilibrium when costs are linear.

It is clear that either the follower excludes the leader by choosing the same location and pricing at $p-\epsilon$, or he accommodates with an opposite kind of product. There is thus maximal differentiation in any equilibrium where the leader makes a strictly positive profit. In this case, the circular market can be seen as two separate linear markets with firms located at the extreme points. The indifferent consumer is such that: $p+t y=q+t(1-y)$, and thus $y=\frac{q-p+t}{2 t}$. Moreover it should be the case that $y>0$ if the leader makes a profit, thus one should have: $p<q+t$.

The follower maximizes $q(1-y)=q \frac{t+p-q}{2 t}$. The first order condition implies: $q=\frac{p+t}{2}$, and consequently $\Pi_{B}=\frac{(p+t)^{2}}{8 t}$.

Firm A maximizes $p \frac{3 t-p}{4 t}$ under the constraints $p<3 t$ (positive market share) and $p \leq \frac{(p+t)^{2}}{8 t}$ (the follower prefers accommodation to exclusion).

The second constraint is the binding one and we obtain the equilibrium values $p=(3-2 \sqrt{2}) t$ and $q=(2-\sqrt{2}) t$. Profits are: $\Pi_{A}=\left(3 \frac{\sqrt{2}}{2}-2\right) t$ and $\Pi_{B}=\sqrt{2} \Pi_{A}$.

We may notice that whereas there is still maximal differentiation, prices are lower with catalog competition than in the standard timing of Salop

\footnotetext{
${ }^{13}$ Let us point out that, as long as both firms locate at the opposite, any location couple is possible. In addition the simultaneous choice of location exhibits a strong coordination problem.
} 
(1979). In fact, in a linear city, the leader can use both price and product characteristic to prevent exclusion - he can locate away from the center of the market in addition to pricing low. In turn, there is no niche on a circular market -the only way to discourage undercutting is to post a low price. This implies that while locations are the same as in the two-stage (locations, then prices) Nash equilibrium, the price of the leader is smaller. Overall, in equilibrium, the leader is thus using a low price - high market share (more than a half) strategy, while the follower is using a high price low quantity strategy.

\section{Discussion}

\subsection{The relative flexibility of prices}

A feature of the model that deserves attention concerns the relative flexibility of location and price. While in a geographical interpretation, the location choice is a long-term decision relatively to the price decision, one can think of markets where both variables are equally flexible. The catering industry provides a good example of this. The product lifetime is very short (one day, if the cooks behave properly), and the choice of product can be a daily decision for independent restaurants, making product choice and price choice equally flexible. In contrast, for chains, uniform pricing and centralized decisions imply more inertia. In other words, chains make their price public by advertisement and national catalogs, while independent stores react to these leaders. The converse is obviously not true: local competition by a small competitor do not lead chains to revise their catalog. Going deeper into this example, one can remark that the model also fits the observation that most chain restaurants sell rather specialized products (like pizzas or 
Tex-mex food $\left.{ }^{14}\right)$, at lower prices than small competitors.

Travel agencies on internet provide an illustration of the predicted results in the quadratic costs case. Typically common travel agencies were rather specialized in a niche (as in d'Aspremont et al. (1979)) such as a continent destination or a category ranking of packages. Internet changed the market positioning strategies of firms because the choice of product is more flexible on internet. Indeed software routines can recombine a package bundling hotels and flights almost instantly, and information technologies have at the same time provided access to large databases of offers that companies use dynamically to redesign products. Therefore firms seem now to compete in a way comparable to our model. This may explain that differentiation has been reduced and competition is fiercer, in the spirit of our results.

Of course, the conclusions would be affected when considering multiproduct firms. It would clearly be worth inquiring the full catalog competition problem with product line differentiation (see Monteiro and Page (2008) for the most recent contribution on that topic).

\subsection{A business strategy interpretation}

In our sequential setting we proved that the follower always prices higher and locates exactly where the indifferent consumer is in equilibrium. On linear market, the leader's strategy appears therefore as a low price strategy a low market share strategy. This type of strategies is described in Gelman and Salop (1983). In their model, the strategic choices are also bidimensional but instead of choosing a product/price couple firms choose a capacity/price couple. Leaders choose a low capacity/price couple giving

\footnotetext{
${ }^{14}$ Hamburgers also are considered specialized food in Europe.
} 
rise to low market share. This is what Gelman and Salop (1983) call a 'judo economics' effect. In our model, leaders commit to be small and hence limit the loss of market share due to competition by choosing a specific product exactly as leaders would choose a low capacity in order to not threaten future competition. Moreover they choose a low price in order to make its market unattractive to competition and to limit the followers' willingness to exclude leaders. ${ }^{15}$

Fudenberg and Tirole (1984) call this behavior the 'puppy dog' behavior. In their analysis, firms also face a bi-dimensional competition involving an investment choice followed by a strategic choice, such as a price. Here the price decisions are strategic complements and a central location choice appears as tough. The same conclusion therefore holds: the leader uses a 'puppy dog' behavior by choosing a specific product and in order to protect its own market it uses a low price strategy.

Interestingly, the equilibrium strategies in the circular setting are different. The leaders cannot anymore use the location strategy to pacify competition: a specialized niche market does not exist. In other words, the leader's only means to appear soft would be to use a high price strategy. But this strategy precisely makes undercutting at the same location very attractive to the follower. Hence this induces leaders to be actually more aggressive in prices, to make undercutting unprofitable. As a result, in the circular market, the leader's strategy is 'top dog' behavior, as described in Fudenberg and Tirole (1984).

\footnotetext{
${ }^{15}$ Gelman and Salop (1983) note p.319 that the judo economics story could also hold with differentiated products: choosing a specialty product is akin to choosing a low capacity according to them. We give a precise formal meaning to their claim.
} 


\subsection{On sequential settings with linear costs}

In models of horizontal differentiation, seven possible sequential settings may be considered ${ }^{16}$. We already know that the (three) cases where firms choose prices simultaneously have no equilibrium in pure strategies. Up to our knowledge, Anderson (1987) is the closest paper about sequential prices and differentiation. He studies the two cases (2 and 3 in figure 5) where firms first sequentially choose their location and then sequentially choose their prices with linear costs. He proves that the most likely situation is the one with the location leader being subsequently the price follower (case 3). The explanation is that the location leader can choose a product such that the location follower prefers to be the price leader: the location leader then locates at the center of the market.

The credibility of the equilibrium he favors might be questioned using our timing structure. If the location follower decides to wait for the location leader to post her price before choosing her product (which is our timing structure), he makes a greater profit. Thus if, ex ante, the follower can credibly commit to enter an attrition game until the leader posts a price, then the location leader will post her price before the entry of the follower. However, this attrition strategy is not credible per se: if the leader chooses her strategy as in Anderson's model $(a=0.5)$ then the follower has an incentive to choose his product before the leader posts her price. Otherwise, the location leader would have to post a tiny price in order to prevent undercutting, which would spoil the market for both firms.

Hence, we can distinguish two cases. If the choice of product is irre-

\footnotetext{
${ }^{16}$ We do not consider unlikely cases in which firms choose their prices before the locations.
} 
(1)

Hotelling

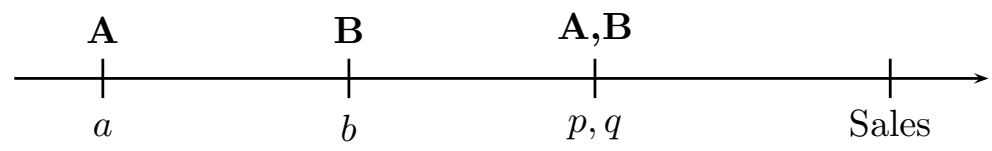

(2)

Anderson I (1987)

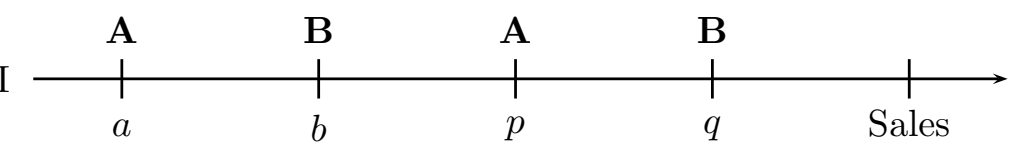

Anderson II (1987)

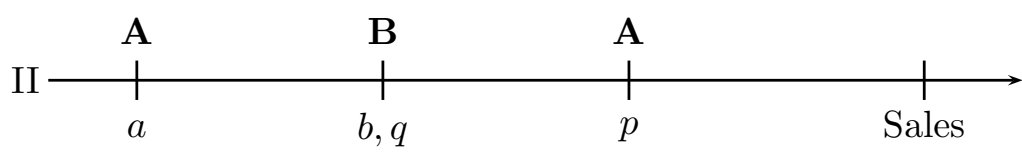

$(4)$

This model

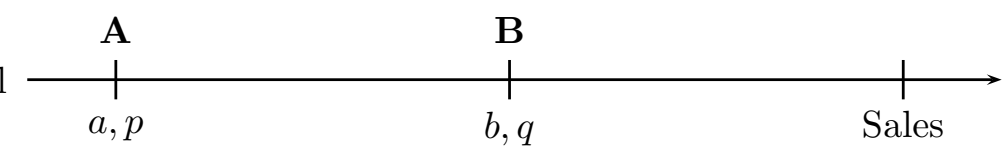

Figure 5: Timing comparison

versible without fixing a price, then Anderson's model is more relevant: the leader chooses a generic product $(a=.5)$, the follower chooses a specific product $(b=0.869)$ and its price, the leader in price is the location follower; prices are relatively high $\left(p_{A}=1.277, p_{B}=1.185\right)$ as well as profits $\left(\Pi_{A}=0.815\right.$ and $\left.\Pi_{B}=0.428\right)$. In this case, the leader is better off than the follower. On the contrary, if the commitment of firms can only be a productprice couple, or if firm B can credibly commit to enter the market only after firm A chose its price, then our model is relevant. The follower has a second mover advantage. The leader chooses a specific product $(a=1 / 9)$ with a low price $\left(p_{A}=0.444\right)$ in order to secure her market while the follower thrives under the umbrella price of the leader while choosing a more generic product $\left(b=.333, p_{B}=0.667\right)$. The profits are then low $\left(\Pi_{A}=0.148\right.$ and $\left.\Pi_{B}=0.444\right)$. This catalog competition is fiercer than the Anderson's one. 
Moreover, there is a value to commit to a product independently of a price only for the leader.

\section{Conclusion}

We have investigated the existence and shape of equilibrium in pure strategies when firms choose at the same time a location and a price in Hotelling's duopoly. There is no equilibrium in the simultaneous game, while when firms successively choose their catalog there exist equilibria with positive profits. An equilibrium is such that: 1) the follower always earns more than the leader, whatever the distribution of consumers and the costs structure; 2) the follower charges a higher price than the leader: the leader in time is the leader in price; 3) in both linear and quadratic cases, the leader makes

the follower indifferent between excluding him from the market and choosing a location such that each firm has a fraction of the market; 4) Differentiation is never minimal nor maximal in equilibrium, and this is qualitatively independent of the costs' shape; 5) The follower may end up with both higher price and higher market shares than the leader.

In the linear cost case, a sequential catalog competition is likely to stabilize the market (there is no pure strategy equilibrium otherwise), while if costs are quadratic or when the market is circular, catalog competition harms the firms. This might explain why on some markets firms mostly communicate on the characteristics of their products but do not post prices. Moreover, in the circular version of the model, the firms use opposite strategies: one chooses a high volume strategy, and the other chooses a high price strategy.

There exist many markets where the choice of product is flexible and 
where consumers get information only through advertisement and catalog, and as such activities are costly, firms do not continuously undertake them. This suggests that the dynamics of prices should also be analyzed in terms of sequentiality of announces and heterogeneous reactive abilities in horizontal differentiation models.

\section{A Proofs}

\section{A.1 Proof of proposition 2:}

We assume that consumers are spread over the market according to some given density function $f(x)$. We consider the case of a finite willingness to pay $v$. The case of infinite willingness to pay is obtained in a similar (and even simpler) way.

Assume that $\Pi_{B}<\Pi_{A}$ in a subgame perfect equilibrium, denote the corresponding strategies by $\left(a^{*}, p^{*}\right)$ and $\left(b^{*}, q^{*}\right)$ and set $\Delta=\Pi_{A}-\Pi_{B}>0$. We show that firm B is better off by choosing catalog $\left(a^{*}, p^{*}-\epsilon\right)$.

Let $S$ be the set of consumers served by firm A in such an equilibrium:

$$
S=\left\{x \mid v-C\left(x, a^{*}\right)-p^{*} \geq 0 \text { and } C\left(x, a^{*}\right)+p^{*} \leq C\left(x, b^{*}\right)+q^{*}\right\}
$$

and let $\mu$ be its weight: $\mu=\int_{S} f(x) d x$. By definition of S, $\Pi_{A}=p^{*} \mu$. Let $S^{\prime}$ be the set of consumers served by firm A if firm B was not here:

$$
S^{\prime}=\left\{x \mid v-C\left(x, a^{*}\right)-p^{*} \geq 0\right\} \supset S
$$

For any $\epsilon>0$, by setting $(b, q)=\left(a^{*}, p^{*}-\epsilon\right)$, firm B clearly attracts all consumers in $S^{\prime}$. Since $S \subset S^{\prime}$, he gets then a profit at least equal to $(p-\epsilon) \mu=\Pi_{A}-\epsilon \mu$. For $\epsilon<\frac{\Delta}{\mu},(p-\epsilon) \mu>\Pi_{A}-\Delta=\Pi_{B}$. This contradict 
the fact that $\left(b^{*}, q^{*}\right)$ is an equilibrium strategy. Thus in any equilibrium one has necessarily $\Pi_{B} \geq \Pi_{A}$.

\section{A.2 Proof of lemma 1}

There remains to consider zones III and III' of figure 2. The follower wants to minimize $y$ because its market share consists of the clients to the right of $y$. By definition, $y$ is implicitly given by: $p+C(y-a)=q+C(b-y)$, for all $b$ such that the solution is in $[0,1]$. If such $y$ does not exist, then the follower has no market share (recall $q \geq p$ in these zones), and we can discard this strictly dominated case. Differentiating the implicit definition of $y$ with respect to $b$, we have:

$$
\left[C^{\prime}(y-a)+C^{\prime}(b-y)\right] \frac{\partial y}{\partial b}=C^{\prime}(b-y)
$$

. In zone III, one has $a \leq y \leq b$. Thus $C^{\prime}(y-a)+C^{\prime}(b-y) \geq 0$ and $C^{\prime}(b-y) \geq 0$, therefore $y$ is increasing in $b$, and the follower wants to set $b$ as small as possible.

In zone III', one has $a \leq b \leq y$, thus $y-a \geq y-b$. Then by convexity of $C, C^{\prime}(y-a)+C^{\prime}(b-y) \geq 0$. Since $b-y \leq 0, C^{\prime}(b-y) \leq 0$, and overall $y$ is thus decreasing in $b$. Therefore in zone III' the follower wants to set $b$ as large as possible.

Since $y$ is continuous in $b$, we can conclude that it must be the case that $b=y$ at an optimal accommodation strategy. Finally, $q=p+C(b-a)>p$ by substituting in the definition of $y$.

\section{A.3 Proof of proposition 3:}

From lemma 1 , we can restrict attention to $y=b$ for accommodation, so that $q=p+C(b-a)$. By symmetry, we assume without loss of generality 
that $0 \leq a \leq \frac{1}{2}$, and clearly $a \leq b$. We consider a uniform distribution of consumers, i.e. $f(x)=1$ for all $x$.

We study the game backwards: the first three steps analyze the follower's program and the last step concerns the leader's optimization. The accommodation profit of the follower is:

$$
\Pi_{B}^{A c}=q(1-y)=[p+C(b-a)](1-b)
$$

The first and second order conditions are:

$$
\begin{array}{cc}
(F O C) & p+C(b-a)=(1-b) C^{\prime}(b-a) \\
(S O C) & (1-b) C^{\prime \prime}(b-a)-2 C^{\prime}(b-a) \leq 0
\end{array}
$$

Step 1: Uniqueness of the interior solution in case of existence.

From condition 1, the SOC is always satisfied. Moreover, the accommodation profit is strictly concave for $b \neq a$. Therefore, for any catalog of the leader, there is at most one $b$ that satisfies the first-order condition.

Step 2: Boundedness of $p$ at an interior solution.

Now we need to check that the optimal $p$ is necessarily finite when there is an interior solution (otherwise it could be the case that there does not exist a best strategy of the leader). We know that a best accommodation strategy necessarily satisfies (FOC), thus

$$
p \leq p+C(b-a)=(1-b) C^{\prime}(b-a) \leq C^{\prime}(1)
$$

. Necessarily $p \leq C^{\prime}(1)$ for accommodation.

Step 3: The exclusion constraint implies the existence of an interior solution.

We want to show that when the leader prevent exclusion, then necessarily 
there exists some $b^{A c}$ a strict interior solution to the follower maximization. By excluding the leader, the follower can get a profit as close as desired to $p$. So in order to prevent exclusion, the leader must choose a catalog such that the accommodation strategy yields a profit greater than or equal to $p$ :

$$
[p+C(b-a)](1-b) \geq p
$$

for at least one $b$ along the accommodation curve defined in lemma 2 . If there does not exist an interior solution then the best accommodation strategy is such that either $b$ is equal to 1 which yields to zero profit or $b$ is equal to $a$. Thus the accommodation profit is $(1-\lambda) p$, which is lower than the exclusion profit since exclusion yields as close as desired to $p$. Therefore, under the exclusion constraint, there exists a strictly interior optimal accommodation strategy. We know that this accommodation strategy is unique from step 2.

\section{Step 4: The Leader's optimization.}

We have seen in step 2 that the corresponding domain is bounded since it is included in $[0,1] \times\left[0, C^{\prime}(1)\right]$. Step 3 tells us that, on this domain and under the exclusion constraint, an interior accommodation strategy always exists and is uniquely defined as a function of $(a, p)$. The program can thus be stated as:

$$
\max _{a, p} \Pi_{A}^{A c}=p b^{A c}(a, p)
$$

under the constraints:

$$
\begin{gathered}
b^{A c}(a, p)=\operatorname{Arg} \max _{a \leq b \leq 1}\left\{\Pi_{B}^{A c}(b, a, p)=(1-b)(p+C(b-a))\right\} \\
\left(1-b^{A c}(a, p)\right)\left(p+C\left(b^{A c}(a, p)-a\right)\right) \geq p \\
0 \leq a \leq \frac{1}{2} \quad 0 \leq p \leq C^{\prime}(1)
\end{gathered}
$$

The function $\Pi_{B}^{A c}$ is a differentiable function of $(b, a, p)$. We have seen that under the exclusion constraint there exists a unique interior solution for the 
maximum problem for all $(a, p)$ thus $b^{A c}(a, p)$ is continuous. Thus $\Pi_{A}^{A c}$ is a continuous function. The domain is non empty: consider for example $a=0$ and $0<p<C\left(\frac{1}{2}\right)$, then the follower prefers $b=\frac{1}{2}$ to exclusion because $p<\frac{1}{2}\left(p+C\left(\frac{1}{2}\right)\right)=\Pi_{B}^{A c}$. This shows that on this domain the leader obtains strictly positive profits. The constraints are not strict and the domain is bounded thus it is a non-empty compact. This ensures that the leader's program has a solution. Therefore, there exists a subgame perfect equilibrium to the sequential catalog game with a strictly positive profit for both firms.

\section{A.4 Proof of proposition 5:}

The proof follows the lines of proposition 3, although condition 1 does not apply. The indifferent consumer y is such that $p+t(y-a)^{2}=q+t(y-b)^{2}$. There is a unique solution such that $y \in[a, b]$. Moreover, we know that the best reply of the follower must be such that $y=b$, thus $q=p+t(b-a)^{2}$. We first characterize the best accommodation strategy. The follower's accommodation program is:

$$
\left(P_{A c}\right) \quad \max _{q, b} q(1-b)=\max _{b \in[a, 1]}\left(p+t(b-a)^{2}\right)(1-b)
$$

When the solution of $\left(P_{A c}\right)$ is not interior, this means that the follower uses an exclusion strategy. Indeed, a corner solution is either $b=1$ but then this would mean zero profit for the follower, and it is therefore impossible, or $b=a$ and $q=p$, but this is dominated for the follower by exclusion, with $b=a$ and $q=p-\epsilon$ for $\epsilon$ smaller than $\lambda$. Thus the leader should act such that the solution of $\left(P_{A c}\right)$ is interior to make a profit.

First order condition implies: $-3 b^{2}+b(4 a+2)-p / t-a^{2}-2 a=0$. The discriminant is $\Delta=4\left[(a-1)^{2}-3 p / t\right]$, so the leader must use a strategy 
such that $p \leq t \frac{(a-1)^{2}}{3}$ in order not to be excluded by the follower.

We have two solutions in $b$, and the second order condition is $2(2 a+1-$ $3 b)$ : The only maximum is $b^{*}(a, p)=\frac{1}{3}\left[1+2 a+\sqrt{(a-1)^{2}-3 p / t}\right]$.

However, the leader must still guarantee a profit of $p$ to the follower in order to prevent an exclusion strategy.

The leader maximizes: $\max _{a, p} p b^{*}(a, p)=p \frac{1+2 a+\sqrt{(a-1)^{2}-3 p / t}}{3}$ under the constraint that the follower accommodates: $p b \leq t(b-a)^{2}(1-b)$

Let us ignore the other constraints $\left(0 \leq a \leq \frac{1}{2}\right.$ and $\left.a \leq b \leq 1\right)$ and check them at the end. Maximizing first in $a$ and then in $p$ yields:

$$
\frac{\partial \Pi_{A}}{\partial a}=\frac{p}{3 t}\left[2+\frac{a-1}{\sqrt{(a-1)^{2}-3 p / t}}\right] \text { and } \frac{\partial^{2} \Pi_{A}}{\partial a^{2}}=\frac{p}{3 t}\left[\frac{-3 p / t}{\left[(a-1)^{2}-3 p / t\right]^{3 / 2}}\right]<0
$$

Maximization implies $a=1-2 \sqrt{p / t}$. As $a$ has to be positive, it implies that $p$ must be inferior to $t / 4$.

Either $p \leq t / 4$ and $a^{*}=1-2 \sqrt{p / t}$ or $p>t / 4$ and $a^{*}=0$. But if $a=0$ then the constraint $p b \leq t(b-a)^{2}(1-b)$ implies $p \leq t / 4$. Thus the leader has to choose a strategy such that $p \leq t / 4$.

Let us now consider the leader's program: $\max _{0 \leq p \leq \frac{t}{4}} \Pi_{A}=p-\frac{p^{3 / 2}}{\sqrt{t}}$. On the admissible domain we have $\frac{\partial \Pi_{A}}{\partial p}=1-\frac{3}{2} \sqrt{p / t}>0$.

Finally, we obtain $p^{*}=\frac{t}{4}$ and $a^{*}=0$ and the optimal strategy of the follower is: $\left(b^{*}, q^{*}\right)=\left(\frac{1}{2}, \frac{t}{2}\right)$, this solution respects all the constraints.

\section{References}

Anderson, S., 1987. Spatial competition and price leadership. International Journal of Industrial Organization 5, 369-398. 4, 7, 8, 21

Dasgupta, P., Maskin, E., 1986. The existence of equilibrium in discon- 
tinuous economic games I: theory; II: applications. Review of Economic Studies 53, 1-41. 3, 11

D'Aspremont, C., Gabszewicz, J., Thisse, J.F., 1979. On Hotelling's 'Stability in Competition'. Econometrica 47, 1145-1150. 3, 14, 19

Economides, N., 1984. The Principle of minimum differentiation revisited. European Economic Review 24, 345-368. 3

Economides, N., 1987. On Nash Equilibrium existence and optimality in Oligopolistic Competition in Prices and Varieties. Greek Economic Review 9, 198-209. 7

Economides, N., Howell, J., Meza, S., 2002. Does it Pay to be the First? Sequential Locational Choice and Foreclosure. Working paper, Stern School of Business, New York University. 4

Fleckinger, P., Lafay, T., 2003. Horizontal Differentiation and Price Competition with Sequential Entry, Working paper 2003-23, CECO, Ecole Polytechnique. 8

Fudenberg, D., Tirole, J., 1984. The Fat-Cat Effect, the Puppy-Dog Ploy, and the Lean and Hungry Look, American Economic Review 74, 361 - 66. 20

Gal-Or, E., 1985, First Mover and Second Mover Advantages, International Economic Review, 26, No. 3, 649-653. 8

Gelman, J., Salop, S., 1983. Judo Economics: Capacity Limitation and Coupon Competition, Bell Journal of Economics 14, 315-325. 19, 20 
Götz, G., 2005. Endogenous sequential entry in a spatial model revisited. International Journal of Industrial Organization 23, 249-261. 4

Hinloopen, J., van Marrevijk, C., 1999. On the limits and possibilities of the principle of minimum differentiation. International Journal of Industrial Organization 17, 735-750. 3

Hotelling, H., 1929. Stability in competition. Economic Journal 39, 41-57. 5

Lambertini, L., 1997. Unicity of equilibrium in the unconstrained Hotelling model. Regional Science and Urban Economics 27, 785-798. 4

Lambertini, L., 2002. Equilibrium locations in a spatial model with sequential entry in real time. Regional Science and Urban Economics 32, 47-58. 4

MacLeod, W. B., 1985. On the non-existence of equilibria in differentiated product models. Regional Science and Urban Economics 15, 245-262. 3

Monteiro, P.K., Page, F.H., 2008. Catalog competition and Nash equilibrium in nonlinear pricing games. Economic Theory 34, 503-524. 3, 19

Neven, D.J., 1987. Endogenous sequential entry in a spatial model. International Journal of Industrial Organization 5, 419-434. 4

Osborne, M., Pitchik, C., 1987. Equilibrium in Hotelling's Model of Spatial Competition. Econometrica 55, 911-22. 3

Prescott, E.C., Visscher, M., 1977. Sequential location among firms with foresight. Bell Journal of Economics 8, 378-393. 4

Salop, S.C., 1979. Monopolostic Competition with Outside Goods. Bell Journal of Economics 10, 141-156. 5, 16, 17 
Tabuchi, T., Thisse, J.F., 1995. Asymmetric equilibria in spatial competition. International Journal of Industrial Organization 13, 213-227. 4 